\title{
Caracterización de la candidiasis vulvovaginal en mujeres de la ciudad de Medellín, Colombia
}

\author{
Clara Duque', Beatriz Gómez ', Olga Uribe' \\ Juan Alarcón', Felipe Soto', Luz Uran', Saray Montiel' \\ Grupo Investigación Biociencias, Institución Universitaria Colegio Mayor de Antioquia \\ Correspondencia: andresnatalia@une.net.co \\ Recibido: 08-08-09 / Aceptado: 25-11-09
}

\begin{abstract}
Resumen
La candidiasis vulvovaginal afecta la mucosa vaginal y es causada por diferentes especies del género Candida. Las pacientes se quejan principalmente de escozor y dolor. El objetivo de esta investigación fue caracterizar la candidiasis vulvovaginal en un grupo de mujeres con vaginitis. Se incluyeron, previo consentimiento informado, 150 pacientes con diagnóstico de vaginitis por Candida entre febrero de 2006 y noviembre de 2008. Se tomó una muestra de secreción vaginal para directo y cultivo. La identificación de especie se confirmó por el método API 20 C AUX, y el perfil de sensibilidad por ATB fungus. La prevalencia de las diferentes especies de Candida fue de: C. albicans 80\% (120), C. parapsilosis 10\% (15), C. glabrata 5,3\% (8), C. tropicalis 2\% (3), C. guillermondii 1,3\% (2), C. kefyr 0,7\% (1) y Candida famata $0,7 \%(1)$.

El 90\%, 135 de ellas, manifestaron síntomas asociados a infección por Candida spp; el 44,5\%, 60 mujeres, presentaron simultáneamente prurito y flujo vaginal. El 6\% de las pacientes presentó más de 4 episodios de candidiasis vulvovaginal en un año. El 90\% de los aislamientos de Candida albicans fueron sensibles al fluconazol, itraconazol y voriconazol. El I00\% de los aislamientos de C. glabrata sensibles a fluconazol y voriconazol. C. kefyr fue intermedio a fluconazol en el 100\% de los aislamientos. Todas las otras especies aisladas fueron 100\% sensibles a los antimicóticos evaluados. C. albicans fue la levadura más frecuentemente recuperada, seguida de C. parapsilosis y C. glabrata. La sensibilidad en general a los antimicóticos es alta para todos los aislamientos.
\end{abstract}

Palabras clave: antimicóticos, candidiasis, Candida, vaginitis.

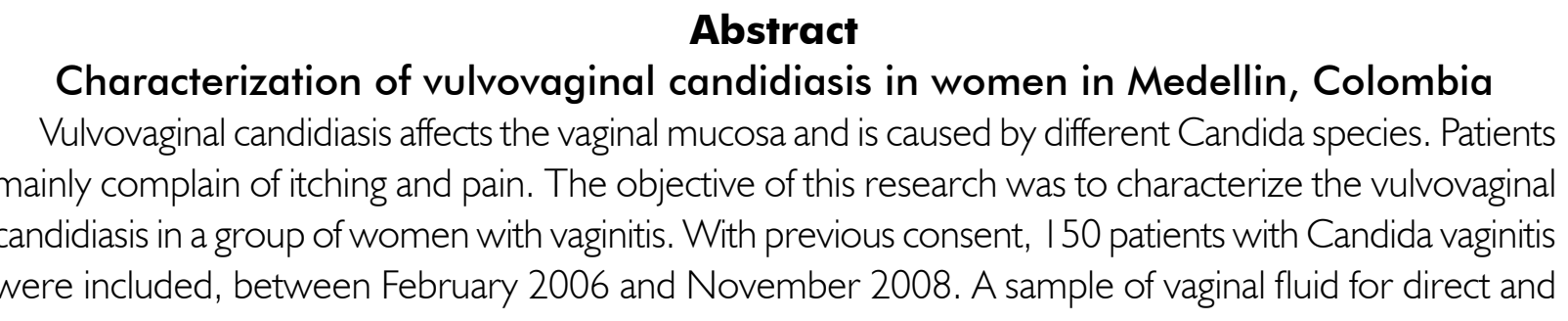


culture was taken. Species identification was confirmed by the API 20 C AUX method, and the sensitivity profile of ATB fungus. The prevalence of different species of Candida was: C. albicans 80\% (I20), C. parapsilosis 10\% (I 5), C. glabrata 5.3\% (8), C. tropicalis 2\% (3), C. guillermondii I.3\% (2), C. kefyr 0.7\% (I), and Candida famata $0.7 \%(\mathrm{I})$.

Of all women, 90\% (135 of them) showed symptoms associated with infection by Candida spp; $44.5 \%, 60$ women, showed simultaneous itching and vaginal discharge. $6 \%$ of patients had more than 4 episodes of vulvovaginal candidiasis in a year. $90 \%$ of Candida albicans isolates were susceptible to fluconazole, itraconazole and voriconazole. $100 \%$ of the isolates of C. glabrata susceptible to fluconazole and voriconazole. C. kefyr was intermediate to fluconazole in 100\% of the isolates. All other species were $100 \%$ susceptible to the antifungals tested. C. albicans was the yeast most frequently recovered, followed by $C$. parapsilosis and C. glabrata. The general sensitivity to the antifungal is high for all isolates.

Keywords: antifungal, Candida, candidiasis, vaginitis.

\section{Introducción}

La candidiasis vulvovaginal (CVV) afecta la mucosa vaginal y la vulva, es causada por diferentes especies del género Candida. Las pacientes se quejan principalmente de escozor y de dolor. El flujo suele ser inodoro y de color blanco amarillento. Los síntomas se intensifican en ambientes húmedos. Las manifestaciones clínicas comprenden enrojecimiento y tumefacción inflamatorias de la vulva y la vagina. A menudo se ven signos de rascado como fisuras y erosiones. Pueden desarrollarse placas blancas de consistencia cremosa en la pared vaginal y en el cérvix (1-5).

Los factores de riesgo asociados a esta infección son: empleo de dispositivo intrauterino (DIU), espermicidas, inicio de relaciones sexuales a temprana edad, uso inadecuado de antibióticos, uso de protectores diarios, embarazo o presencia de enfermedades de base como la diabetes mellitus $(4,6)$. La principal especie involucrada en la candidiasis es Candida albicans; sin embargo, se ha reportado un aumento en la incidencia de Candida glabrata $(2,3)$. El objetivo de este trabajo fue caracterizar la candidiasis vulvovaginal en un grupo de mujeres con vaginitis, identificar la frecuencia de aislamiento de las diferentes especies, registrar los síntomas clínicos y evaluar la actividad in vitro de los antimicóticos

\section{Materiales y métodos}

En el estudio se incluyeron 150 mujeres con diagnóstico de vaginitis por Candida procedentes de la ciudad de Medellín durante el periodo febrero 2006 a noviembre de 2008. Las muestras fueron remitidas al Laboratorio de investigación de la Institución Universitaria Colegio Mayor de Antioquia (IUCMA) para su posterior análisis. A cada paciente se le realizó encuesta y previo consentimiento informado, se tomaron muestras de flujo vaginal para Gram y cultivo. El cultivo se procesó en Sabouraud Dextrosa Agar y Chromogenic Candida Agar. Todos los aislamientos de levaduras compatibles con Candida $s p$. fueron conservados en $1 \mathrm{~mL}$ de caldo infusión cerebro corazón (BHI) y glicerol al $10 \%$ a $-20^{\circ} \mathrm{C}$.

\section{Identificación de Levaduras}

Al obtener el crecimiento de colonias compatibles con Candida spp. se procedió a practicar la prueba de tubo germinal. Las levaduras fueron identificadas como Candida albicans cuando presentaron tubo germinal y pigmento verde en el agar cromogénico. Aquellos aislamientos donde no se pudo determinar la especie, fueron identificados por el método del API $20 \mathrm{C} \mathrm{AUX}^{\oplus}$ siguiendo las especificaciones del fabricante (bioMérieux, Paris, France). 


\section{Sensibilidad a los antimicóticos}

El método utilizado fue el ATB fungus ${ }^{\mathrm{TM}}$ (bioMérieux, Paris, France), siguiendo las recomendaciones del fabricante, el cual permite determinar la sensibilidad de Candida spp. frente a antimicóticos $(7,8)$ en medio semisólido en condiciones muy próximas a las de la técnica de referencia de microdilución (según las recomendaciones de EUCAST (9) y del Clinical and laboratory Standards Institute (CLSI) (10). Las levaduras fueron identificadas con prueba de tubo germinal, crecimiento en agar cromogénico y asimilación de carbohidratos por el método comercial API 20 C AUX. El método utilizado para evaluar la sensibilidad a los antimicóticos fue el ATB fungus (bioMérieux).

\section{Implicaciones éticas}

El trabajo de investigación fue aprobado por el comité de investigaciones de la IUCMA. A cada paciente se le informó del estudio, se le invitó a formar parte y se le garantizó que los resultados eran confidenciales. Posterior a esto cada paciente firmó su consentimiento informado.

\section{Resultados}

La edad promedio de las pacientes fue de 25 años (14-51 años), los síntomas más frecuentemente fueron el prurito y el aumento de flujo vaginal en forma simultánea. De las 150 pacientes, $135(90 \%)$ manifestaron síntomas asociados a infección por Candida spp; 60 (44.5\%) presentaron simultáneamente prurito y flujo vaginal. La frecuencia relativa de las diferentes especies de Candida aisladas puede observarse en la Figura 1.

El 6\% de las pacientes presentó más de 4 episodios de CVV al año. Al analizar la correlación entre el examen directo y el cultivo se observaron blastoconidias y seudohifas en $117(78 \%)$ de 150 muestras. No se observaron hongos en $33(22 \%)$. En cuanto al perfil de sensibilidad a los antimicóticos se observó que el $90 \%$ de los aislamientos de Candida albicans fueron sensibles al fluconazol, itraconazol y voriconazol, el $10 \%$ intermedio a fluconazol y resistente a voriconazol e itraconazol. El 100\% de los aislamientos de C. glabrata sensibles a fluconazol y voriconazol y el $50 \%$ resistente a Itraconazol. C. kefyr fue intermedio a fluconazol en el $100 \%$ de los aislamientos. Todas las otras especies aisladas fueron $100 \%$ sensibles a los antimicóticos.

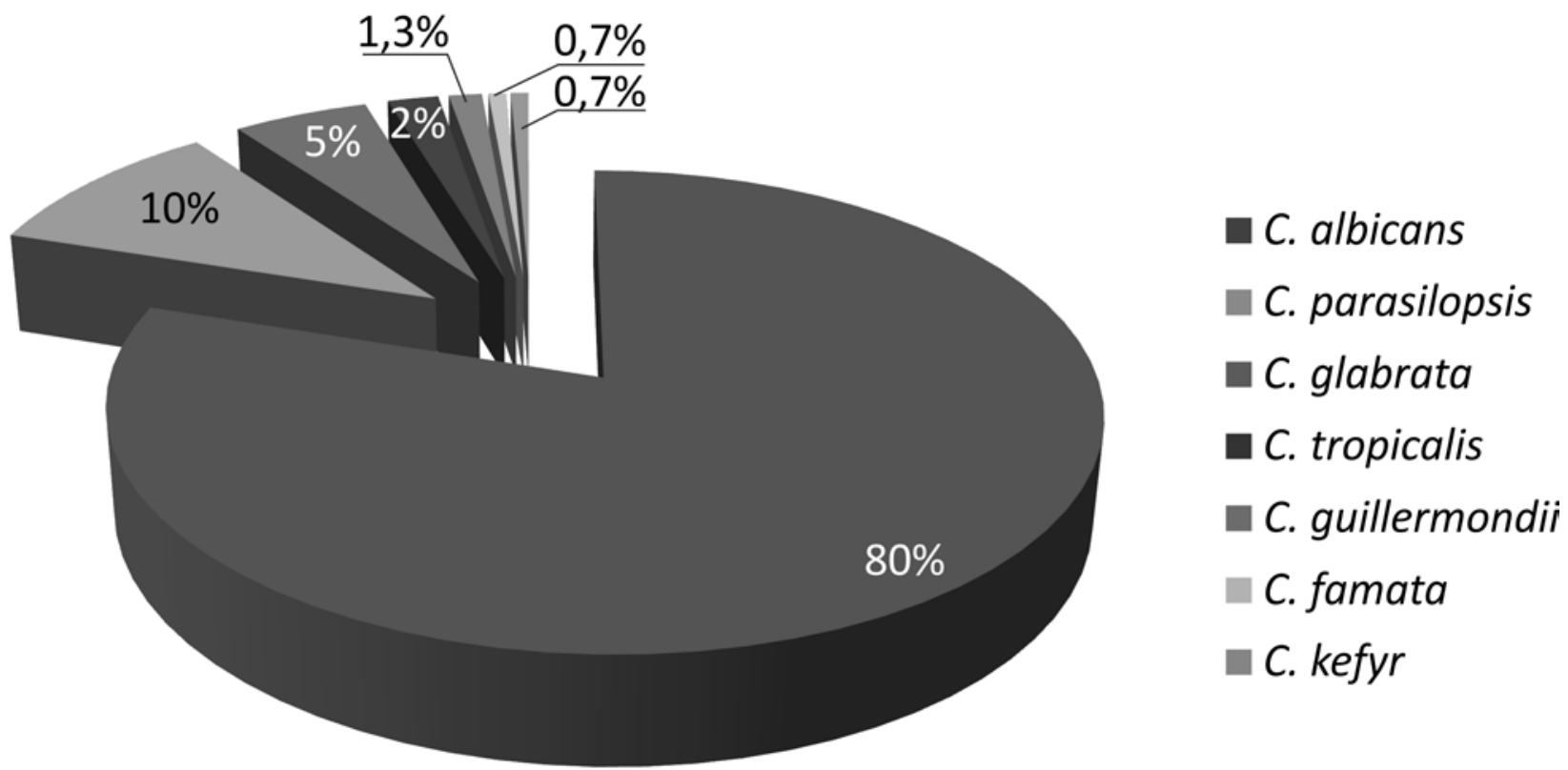

Figura 1. Frecuencia de especies de Candida. La prevalencia de las diferentes especies de Candida fue de C. albicans $80 \%$, C parapsilosis 10\%, C. glabrata 5.3\%, C. tropicalis $2 \%$, C. guillermondii $1.3 \%$, C. kefyr $0.7 \%$ y Candida famata $0.7 \%$. 


\section{Discusión}

En este estudio los síntomas más frecuentemente asociados a la CVV estuvieron en concordancia con los que se reportan en la literatura, siendo el ardor el de menor proporción. Al igual que en otros trabajos, en este estudio $C$. albicans también fue la especie más frecuentemente aislada, seguida por patógenos emergentes como C. parapsilosis, C. glabrata y $C$. tropicalis $(3-5,8,11)$.

Al ser Candida albicans la especie más frecuentemente aislada, es importante emplear en la identificación de las especies métodos moleculares que permitan diferenciar entre C. albicansy C. dubliniensis, ya que los métodos microbiológicos convencionales no permiten realizar dicha diferenciación (12). Los métodos comerciales de identificación de levaduras como Api 20 CAUX son importantes para clasificar el género Candida como agente causal de vulvovaginitis, pues las levaduras del genero Trichosporon pueden producir esta patología en algunas ocasiones (13). Las levaduras del género Candida son sensibles a los antimicóticos; en la población estudiada se observó que el 90\% de los aislamientos de Candida albicans fueron sensibles al fluconazol, itraconazol y voriconazol. Al igual que en el estudio de Torres y Alvarado se concluyó que ATB Fungus es un método sencillo y reproducible para la determinación de resistencia a los antimicóticos en levaduras del género Candida (14). Es de resaltar que el 6\% de las pacientes presentó más de 4 episodios de CVV al año, lo cual define una candidiasis vulvovaginal recidivante. Por lo tanto en este grupo de pacientes es importante investigar factores predisponentes y pruebas de sensibilidad a los antimicóticos (15).

Para terminar, en el examen directo se observaron blastoconidias y seudohifas en $78 \%$ de las muestras, encontrando una sensibilidad similar a la del estudio realizado por
Buitrón y colaboradores, donde el examen directo fue positivo en $60.8 \%$ de los casos. Lo cual ratifica la importancia de este examen en el diagnóstico de candidiasis vulvovaginal (16).

\section{Referencias}

1. Arango M, Castañeda E. Micosis humanas. $2^{\circ}$ edición. Medellín: CIB; 2003.

2. Díaz FJ, Estrada S, Franco L, Jaramillo JM., Maestre A, Ospina S, et al. Microbiología de las infecciones humanas. $1^{\circ}$. Edición, Medellín: CIB. 2007.

3. Sobel J, Chaim W. Update on treatment of vulvovaginal candidiasis. Rev Iberoam Micol. 1996;13:44-46.

4. Rivero M. Frecuencia de especies de Candida aisladas en pacientes embarazadas con vulvovaginitis. Rev Soc Ven de Microbiol. Caracas. 2003;23:148-152.

5. Buscemi L, Nigrona, R. Estudio de vulvovaginitis agudas en pacientes adultas, sexualmente activas con especial referencia a la candidiasis en pacientes del hospital de infecciosas Francisco J. Muñiz. Rev Iberoam Micol. 2004;21:177-181.

6. García M, García S, Copolillo E, Cora M, Barata A, Vay C, et al. Prevalencia de candidiasis vaginal en embarazadas. Identificación de levaduras y susceptibilidad a los antifúngicos. Rev. Argentina Microbiol. 2006;38:9-12.

7. Bauters TG, Dhont MA, Temmerman MI, Nelis HJ. Prevalence of vulvovaginal candidiasis and susceptibility to fluconazole in women. Am J Obstet. Gynecol. 2002; 187:569-574.

8. Richter SS, Galask RP, Messer SA, Hollis RJ, Diekema DJ, Pfaller MA. Antifungal susceptibilities of Candida species causing vulvovaginitis and epidemiology of recurrent cases. J Clin Microbiol. 2005;43:2155-2162.

9. Cuenca-Estrella M, Arendrup MC, Chryssanthou E, Dannaoui E, Lass-Florl C, Sandven P, et al. Multicenter evaluation of the reproducibility of the proposed antifungal susceptibility testhing method for fermentative yeasts. Subcommitte of the european committee on antimicrobial susceptibility testing (AFST- EUCAST). Clin Microbiol Infect. 2003;13:1018-1022.

10. CLSI. Reference method for broth dilution antifungal susceptibility testing of Yeasts. M27-A2. 2002.

11. Ferrer J. Vaginal candidosis: epidemiological and etiological factors. Int J Gynecol Obstet. 2000;71: S21-7.

12. Hospenthal DR, Beckius ML, Floyd KL, et al. Presumptive identification of candida species other than C. albicans, C. krusei and C. tropicalis with the CHROMagar candida. Ann Clin Microbiol Antimicrob. 2006;5:1.

13. Makela PI, Leaman D, Sobel J. Vulvovaginal trichosporonosis. Infect Dis Ostet Gynecol. 2003;11:131-133.

14. Torres J, Alvarado E. In vitro susceptibilities to yeast using the ATB fungus method, compared with sensitive yeast one and standar CLSI (NCCLS) M27-a2 methods. J Antimic Chemoth. 2007;60:658-661.

15. Enache-Angoulvant, A. Reglas de interpretación de las infecciones por Candida. Acta bioquím Clín Latinoam. La Plata. 2007;4:587-593.

16. Buitrón $\mathrm{G}$, Bonifaz A, Amancio O, et al. Correlación clínico-micológica de la candidiasis vulvovaginal. Ginecol Obstet Mex 2007;75:68-72. 